\title{
Psychological Impact of Covid-19 Issues Appearing on Social Media
}

\author{
Kaseru Anduni ${ }^{1}$, Kustel Diac ${ }^{1}$ \\ ${ }^{1}$ Faculty of Psychology and Educational Sciences, University of Bucharest
}

\begin{abstract}
The effect of disseminating content on social media on people's psychology is discussed in this essay. The position of social media in disseminating content with the aim of informing and supplying the public with the most up-to-date information on covid-19 problems is critical. Over the pandemic, mental wellbeing conditions have worsened as a result of the widespread dissemination of inaccurate knowledge about Covid matters, causing a slew of psychiatric challenges in community. Learning difficulties, mood swing symptoms, somatic complaints, and unnecessary fear triggered by the spread of associated facts covid-19, which seems to be quite frightening and risky. The widespread dissemination of hoaxes about Covid-19 on social media has caused widespread paranoia and terror, leading to panic shopping, which has culminated in skyrocketing costs for essential necessities due to shortages, to the point that surgical masks and hand sanitizers are no longer accessible. It not only causes erroneous beliefs, but it also causes uncertainty and anxiety, as well as affecting people's mental health.
\end{abstract}

Keywords: Covid-19, Social Media, Psychological Impact

Received: January 10, 2021

Revised: February 23, 2021

Accepted: March 4, 2021

\section{Introduction}

The first is, of necessity, the news media as a medium of knowledge, which must not neglect its primary responsibility of educating the people. The education in question entails not only presenting longitudinal statistics on COVID-19 victims, but also communicating research and health care to the general population.

The media also has a major impact on consumer attitudes and health-related actions. Meanwhile, although they got vaccine-related material from health publications or clinics, they had varying reactions. As a result, the media's ability to communicate content that educates the public about COVID-19 would have an effect on public health decision.

Synergy and teamwork might be the best ways to describe the magnitude of Indonesia's problems. Difficult will it be for the media to intervene in this case without the permission of the government and the Indonesian people In the case of widespread COVID-19-related apprehension in the population, the government must take both short- and long-term steps.

In terms of short-term solutions, the government, especially via the Ministry of Communication and Intelligence, must be at the forefront of reporting on COVID-19 as the highest information holder. The government must publish material on its own official website. As a result, the society has at least one credible knowledge outlet. Furthermore, group distress caused by false news may be minimized. The government must also be transparent in publishing with a notice.

In the meantime, the government would promote mass media literacy as a long-term remedy. For instance, putting together a media literacy program. This is true not only in the case of the COVID-19 epidemic, but in a variety of other cases as well. The Indonesian public does not seem to be equipped for the accelerated development in information technology. With just 
rudimentary intelligence, Indonesians are prone to accepting any information that is shown to them.

This knowledge can be formed not only through the curriculum, but also through innovative social networking initiatives to diverse segments of society. In addition, lectures and workshops on this subject are becoming more popular in universities. Don't be afraid to collaborate with other media outlets to get something to the public's notice.

One thing is certain: in the aftermath of the COVID-19 pandemic's uncertainty, let's work hard to avoid making matters worse. Instead of seeing something at face value, we don't investigate and discover the facts first. Similarly, as we wish to communicate knowledge to someone, we must ensure that the information is accurate.

The media could therefore contribute to public optimism. The most up-to-date material must be communicated. But, in the press, let's keep it balanced. A rise in the amount of patients classified as hopeful, for example, should be replaced by an increase in the number of patients who have healed. In its news, the media must be willing to reduce negative stigma and bigotry. As COVID-19 was first deemed a pandemic by the World Health Organization (WHO), everybody panicked. Furthermore, the corona virus is doing the rounds in the newspapers and on the television at the same time. Starting with individuals afflicted with a virus whose distribution is so rapid that it will cause victims to die in a brief period of time, the virus's spread is very fast and by direct communication, and other bad news.

\section{Psychological Problems Due to Covid Issues on Social Media}

Children and teenagers, who made up the majority of the participants ( 27.2 percent), faced barriers due to academic problems. Furthermore, stress complaints are common psychiatric issues that affect people of all ages, with a prevalence of 23.9 percent. Anxiety complaints this one psychiatric disorder, including depression complaints, affects people of all ages, with $18.9 \%$ of people reporting it.

Mood shifts are a common complaint. Mood fluctuations or mood swings in the wake of the pandemic is experienced by people of all ages, with a $9.1 \%$ prevalence. Anxiety disorders this anxiety condition is not only a complaint; it may also mean that the participants' emotions of worry, anxiety, or fear are high enough to interfere with their everyday lives, and the figure approaches $8.8 \%$.

Somatic symptoms are an illness in which an individual has discomfort in his body about which there is no medical explanation, and this neurological disorder affects 4.7 percent of the population (Frisoni et al., 2000; Butler et al., 2002). Looking at the presentations received, we partnered with a variety of groups, including the Scout National Quartier with an early psychology service training program.

\section{Covid-19 Issues in Social Media on Community Psychology in the Pandemic Era}

The newspaper, for example, attempts to advertise names and agencies for victims while focusing on COVID-19 victims. "Corona Bank workers indicated Corona," for example, according to the headline. Fear and cynical media speculation regarding this organization occurred instead of commemorating the public who might have worked with the victim. In this coverage, the reporting provided by the newspapers often continues to speak about statistics and case studies. Some news has underestimated the importance of both sides of the cover, whether motivated by the psychology of the journalist who is still scared. The spread of fear in the population is caused by a mixture of exciting names, uneducative news material, and a lack of literacy culture in Indonesian society. 
The news's clickbait phenomenon isn't entirely incorrect. Often a little emphasis in the title is necessary to inform the public of the relevance of a subject. Clickbait seems to be important yet again in order to eradicate the psychological aspect of Indonesian culture, which is illiterate.

The media also associates this outbreak with political and ethnicity, faith, caste, and intergroup problems that are hot in Indonesia. Under such a case, certain mainstream outlets use advantage of the epidemic to stir up problems that defame or discriminate against certain groups.

When the media dubbed COVID-19 the Wuhan virus or the Chinese virus, the negative perception of one of the human races grew. COVID-19 was chosen with great consideration by WHO Director General Tedros Adhanom Ghebreyesus. Of necessity, this is to stop mentioning particular geographic areas, animal populations, or human classes.

Furthermore, the expanding ways of sharing knowledge allow individuals to participate in information distribution. Even if it has a beneficial effect, this phenomenon has the unintended consequence of hastening the spread of reckless knowledge. Hoaxes that spread too widely may trigger general panic or even ignorance to the virus's dangers.

Respecting medical privacy in reporting seems to be a way to minimize inequality that is not just linked to color. The patient's privacy status is being chased by the curious heads of the public in the middle of this mystery. It seems that the media may not need to use the condition in order to increase press attention, but it may have an effect on the public's undue curiosity. The media must remember to place themselves as a form of democratic influence over public policy, just as COVID-19 in this situation. It is proper for the media to band together in support of the greater good. Through such clout, the media will serve as a conduit for encouraging the government to enact laws that benefit the public as well as educating the general public.

\section{Mental Health during the Pandemic}

Several residents in various regions objected to the rapid test being conducted in their neighborhood (Berclaz et al., 2011). Of course, this denial has a history, which includes a shortage of evidence and exposure to hoax information circulated on social media, in comparison to the lack of knowledge accessed. Area is one of the regions that declines to participate in the rapid evaluation. Statements made by reckless individuals and fake news plague society. As a result, culture is concerned.

More than 1,125 fake reports have been discovered on the internet by the Ministry of Information. Turn Back Hoax Covid-19, for example, discovered hoaxes about onions curing Covid-19, cocaine killing the corona, alcoholic drinks and tobacco preventing Covid-19 risk, and the Covid-19 virus being spread through the air.

Because of the widespread distribution of hoaxes about Covid-19 on social media, there was panic purchasing, which culminated in soaring rates for essential necessities owing to shortages, and surgical masks and hand sanitizer were no longer accessible on the market.

This, in addition to causing erroneous beliefs, often causes uncertainty and anxiety, which may have a negative impact on mental wellbeing. For the Covid-19 pandemic, you might imagine. In the current situation of 1,522 people who took part in the survey, 64.3 percent experienced anxiety and depression as a result of COVID-19, particularly when combined with hoaxes related to Covid-19, such as exaggerating information and even making false reports about Covid-19, and several people expressing negative feelings on social media, such as fear, worry, nervousness, anxiety, and so on.

For several countries, the COVID-19 pandemic has evolved into a source of fear, resulting in widespread paranoia. Because of the constant barrage of reports regarding the disease, people's 
psychological and physiological states are being disrupted. As a consequence, pseudo-SARSCoV-2 infection signs such as headache, sore throat, and cough emerge. The COVID-19 pandemic is impacting more than just the general public's emotional wellbeing (Tan et al., 2020; Hu et al., 2020; Maugeri et al., 2020; Mukhtar, 2020; Gualano et al., 2021; Haider et al., 2020). Many citizens are forced to conduct something individually as a result of the physical restraint scheme. Most citizens were weary as a result of "getting sent off." This approach has an effect on cognitive loss / dementia in the elderly, rendering them more vulnerable to fear, frustration, tension, and anxiety. Meanwhile, when the amount of patients exploded at the same moment, health staff had to labor much harder. According to the Centers for Disease Control and Prevention (CDC), this pandemic has the potential to cause mental illness, especially among survivors and health care staff. Depression, fear, and post-traumatic stress disorder are also more likely to occur among them. It has an effect not only on the community's physical fitness, but also on other facets of life. The pandemic has had a significant effect on the emotional wellbeing of many who have been infected. People's lives have changed dramatically since the COVID-19 pandemic began. People find it challenging to respond to abrupt changes, which may lead to depression and trauma.

The stigma attached to survivors and health staff aggravates the situation. The government's sluggish reaction, which ignored the crisis, had a negative impact on the people's mental wellbeing. When the state agreed to implement a federal quarantine, the community's psychological burden increased. At the same period, they must deal with an avalanche of facts from social media, including warnings of PPE shortages, medical personnel shortages, and hospital power shortages in Wuhan. If other countries prepare for the challenge of COVID-19, Indonesia has strengthened its tourism industry. The next example is identical to the CDC's account of the SARS and MERS outbreaks in China.

When the planet is dealing with SARS and MERS outbreaks, there are many patterns of psychiatric illnesses that emerge. Depression, panic attacks, paranoia, suicide, delirium, and psychotic symptoms were all publicly known medical comorbidities.

MERS patients' families must remain segregated from the rest of the world, particularly though they have been pronounced disease-free. SARS survivors displayed symptoms of posttraumatic stress disorder in up to $25 \%$ of cases, and 15.6 percent had severe depression.

Meanwhile, community professionals, who are at the frontline of the fight against the crisis, are often at risk of developing psychiatric illnesses as a result of the double pressure of caring for patients and the social stigma.

This answer was obtained only recently, until things returned to usual. Meanwhile, other coworkers' accounts were no less depressing, since they had to relocate to a boarding house because their workplace was concerned that the infection might spread. Then there were the children of medical professionals, who were still barred from attending for the same purpose.

\section{Conclusion}

For several countries, the COVID-19 pandemic has evolved into a source of fear, resulting in widespread paranoia. Because of the constant barrage of reports regarding the disease, people's psychological and physiological states are being disrupted. As a consequence, pseudo-SARSCoV-2 infection signs such as headache, sore throat, and cough emerge. During the pandemic, the community's emotional wellbeing was compromised as a result of social networking problems that caused citizens to fear and stress excessively. Increased glorification of knowledge on social media, causing issues such as mood fluctuations, somatic complaints, and undue fear as a result of the spread of information about Covid-19, which seems to be very 
frightening and risky, causing people to become paranoid and refuse to socialize, preferring instead to remain at home. This worsens society's depressive situation.

\section{References}

Berclaz, J., Fleuret, F., Turetken, E., \& Fua, P. (2011). Multiple object tracking using k-shortest paths optimization. IEEE transactions on pattern analysis and machine intelligence, 33(9), 1806-1819.

Butler, J. V., Whittington, J. E., Holland, A. J., Boer, H., Clarke, D., \& Webb, T. (2002). Prevalence of, and risk factors for, physical ill-health in people with Prader-Willi syndrome: a population-based study. Developmental Medicine \& Child Neurology, 44(4), 248-255.

Frisoni, G. B., Fratiglioni, L., Fastbom, J., Guo, Z., Viitanen, M., \& Winblad, B. (2000). Mild cognitive impairment in the population and physical health: data on 1,435 individuals aged 75 to 95. The Journals of Gerontology Series A: Biological Sciences and Medical Sciences, 55(6), M322-M328.

Gualano, M. R., Moro, G. L., Voglino, G., Bert, F., \& Siliquini, R. (2021). Monitoring the impact of COVID-19 pandemic on mental health: a public health challenge? Reflection on Italian data. Social psychiatry and psychiatric epidemiology, 56(1), 165-167.

Haider, I. I., Tiwana, F., \& Tahir, S. M. (2020). Impact of the COVID-19 pandemic on adult mental health. Pakistan Journal of Medical Sciences, 36(COVID19-S4), S90.

Hu, Z., Lin, X., Kaminga, A. C., \& Xu, H. (2020). Impact of the COVID-19 epidemic on lifestyle behaviors and their association with subjective well-being among the general population in mainland China: Cross-sectional study. Journal of medical Internet research, 22(8), e21176.

Maugeri, G., Castrogiovanni, P., Battaglia, G., Pippi, R., D'Agata, V., Palma, A., \& Musumeci, G. (2020). The impact of physical activity on psychological health during Covid-19 pandemic in Italy. Heliyon, 6(6), e04315.

Mukhtar, S. (2020). Mental health and emotional impact of COVID-19: applying health belief model for medical staff to general public of Pakistan. Brain, behavior, and immunity.

Tan, B. Y., Chew, N. W., Lee, G. K., Jing, M., Goh, Y., Yeo, L. L., \& Sharma, V. K. (2020). Psychological impact of the COVID-19 pandemic on health care workers in Singapore. Annals of internal medicine, 173(4), 317-320.

Walker, M., \& Kent, A. (2009). Do fans care? Assessing the influence of corporate social responsibility on consumer attitudes in the sport industry. Journal of Sport Management, 23(6), 743-769. 